\title{
ROTAS TECNOLÓGICAS PARA A PRODUÇÃO DE FERROCROMO NO BRASIL: UM ESTUDO DE VIABILIDADE TÉCNICA, ECONÔMICA E FINANCEIRA
}

\author{
Marcelo Rocha' \\ Paulo Soares Figueiredo ${ }^{2}$
}

\section{Resumo}

A indústria brasileira de ferroligas supre $94 \%$ da demanda interna de ferro cromo, no entanto, a intensa competição existente obriga a indústria a buscar melhoria de eficiência operacional na produção do $\mathrm{FeCrAC}$. A principal barreira para a implantação de inovações é a carência de informações sobre as melhores tecnologias e os custos-benefícios a elas associadas para as empresas. Na primeira etapa deste trabalho foram identificadas quatro rotas tecnológicas que produzem industrialmente o $\mathrm{FeCrAC}$. Essas rotas foram submetidas a dois filtros para a definição da rota mais adequada. $O$ presente estudo visa avaliar estas rotas de acordo com critérios técnicos e econômicos. No primeiro filtro, de avaliação técnica, duas rotas foram selecionadas. No segundo filtro, de avaliação econômico-financeira, a rota de operação em forno elétrico fechado apresentou o melhor resultado em todos os indicadores econômicos.

Palavras-chave: Eficiência energética; Ferrocromo; Forno elétrico de redução; Indústria eletrointensiva.

\section{TECHNOLOGICAL ROUTES FOR THE PRODUCTION OF FERROCHROME IN BRAZIL: A STUDY OF TECHNICAL, ECONOMICAL AND FINANCIAL VIABILITY}

\begin{abstract} route showed the best results in all economic indicators.

\section{INTRODUÇÃO}

O setor de ferroligas é considerado uma atividade industrial eletrointensiva. As ferroligas podem ser obtidas por diversos processos. $O$ processo de produção de ferroligas mais empregado envolve a redução carbotérmica em fornos elétricos de arco submerso. Este processo envolve, basicamente, as seguintes etapas: preparação das matérias-primas, fusão e redução das cargas, preparação do produto. Dentre as etapas mencionadas, a fusão redutora é a
\end{abstract}

The Brazilian ferroalloy industry supplies $94 \%$ of the domestic demand for ferrochrome. However, the intense competition existing forces the industry to seek improvement of operational efficiency in the production of FeCrAC. The major barrier to the deployment of innovations is the lack of information on the best technologies and the cost benefit related to them for the companies. In the first step of this study, four technological routes to produce $\mathrm{FeCrAC}$ on an industrial scale have been identified. These routes were submitted to two filters to define the most suitable route. This study aims to evaluate these routes according to technical and economic criteria. In the first filter, technical evaluation, two routes were pre-selected. In the second filter, economic and financial valuation, the closed electric furnace operation

Keywords: Energy efficiency; Ferrochrome; Submerged arc furnace; Electrointensive industry.

mais importante, tanto do ponto de vista de produção física quanto sob o aspecto do consumo de energia [I]. De acordo com Leite et al. [2], o maior potencial está associado à etapa de fusão e redução da carga $-80 \%$ do total - em fornos elétricos. Este potencial é relativo ao consumo energético.

A eficiencia de alguns processos podem ser melhorados pelo adequado processamento parcial da matéria prima antes da mesma entrar no forno elétrico de redução. Isso pode

'FERBASA, Salvador, BA, Brasil.

${ }^{2}$ Departamento de Modelagem Computacional, SENAI CIMATEC, Salvador, BA, Brasil. E-mail: paulo_sfigueiredo@hotmail.com

2176-1523 (C) 2016 Associação Brasileira de Metalurgia, Materiais e Mineração. Publicado pela ABM. Este é um artigo de acesso aberto distribuído sob os termos da licença Creative Commons CC BY-NC-ND (Attribution-NonCommercial-NoDerivs) - https:// creativecommons.org/licenses/by-nc-nd/4.0\%. 
ser alcançado a partir de três processos: aglomeração dos finos, pré-redução do minério e pré-aquecimento das matérias primas.

Embora haja uma certa variedade de rotas tecnológicas, existem provavelmente quatro grupos que cobrem praticamente todos os processos para a produção do $\mathrm{FeCrAC}$. De acordo com Gasik [3], os grupos são os seguintes:

I. Forno elétrico a arco submerso aberto e semi fechado.

2. Forno elétrico a arco submerso fechado, com ou sem pré-aquecimento.

3. Pré redução seguida por forno elétrico a arco submerso fechado.

\section{Forno a arco DC (corrente contínua) aberto.}

A seleção da rota tecnológica é determinada por um conjunto de fatores, tais como: acesso a uma determinada tecnologia, caracteristica e disponibilidade de matérias primas (principalmente minério de cromo), custo da eletricidade, eficiência metalúrgica, eficiência elétrica, economia de escala, investimento de capital e legislação ambiental e saúde ocupacional.

A tecnologia adotada atualmente na indústria brasileira de ferro cromo ( $\mathrm{FeCr}$ ) é o forno elétrico aberto convencional, sendo que consumo específico de energia está numa faixa de 4.000 a $4.200 \mathrm{kWh} / \mathrm{t}$. A contribuição típica da energia no custo total de produção nas plantas de ferro cromo é de $22 \%$ e sua contribuição no custo variável é de cerca de $33 \%$, sendo assim, uma variável que afeta diretamente a competitividade dessas plantas [4].

A indústria de ferroligas brasileira supre $94 \%$ da demanda interna de ferro-cromo. $O$ cromo é um elemento insubstituível na produção do aço inoxidável, sendo essa a sua principal aplicação. Isso faz com que este setor da indústria tenha um papel estratégico para a siderurgia nacional.

A FERBASA é uma empresa integrada de ferroligas de cromo e silício, com mais de 50 anos de atuação no mercado siderúrgico. Líder em seu segmento, está entre as 500 maiores empresas do país. É a única produtora de ferroligas de cromo das Américas.

Em março de 2012, a Ferbasa, detentora de 85\% das reservas brasileiras, anunciou em fato relevante que - montante saltou de 13 milhões para 40 milhões de toneladas. Em cálculos simples, este volume corresponde a aproximadamente 100 anos da necessidade da companhia considerando a capacidade de produção atual. Isso significa que a empresa tem reservas de minério de cromo que suportam investimento intensivo em capital para a produção do $\mathrm{FeCrAC}$.

Os custos crescentes de energia elétrica e, portanto, a necessidade de reduzir a energia necessária para a produção de $\mathrm{FeCrAC}$ têm estimulado as inovações nas tecnologias utilizadas para a fusão redutora do minério de cromita. A indústria enfrenta desafios evidentes, como a tendência de queda dos preços de venda e o aumento da consciencia sobre as condições de trabalho e ambientais. Esses desafios podem ser respondidos por tecnologias inovadoras já existentes e pelo desenvolvimento de novos processos.

Embora exista conhecimento sobre estratégias governamentais, atitudes gerenciais e alternativas tecnológicas para a utilização mais racional e eficiente da energia, nos mais diversos processos produtivos, observa-se que são muitos os desafios e barreiras que impedem um avanço mais veloz nos níveis de eficiência energética que são possíveis de serem alcançados. Dentre as diversas barreiras e desafios envolvendo as abordagens da inovação tecnológica para o desenvolvimento mais acentuado dos conceitos e práticas da eficiência energética no Brasil, tem-se: desconhecimento das vantagens econômicas e ambientais da conservação e uso racional da energia em inúmeros setores da atividade produtiva, carência de informações sobre as melhores tecnologias e os custos-benefícios a elas associados para as empresas, difícil avaliação dos resultados econômicos alcançados pelas empresas, seja pela dificuldade de se calcular os ganhos ou pela falta de percepção dos reais benefícios previstos.

O objetivo deste estudo é definir a rota mais apropriada a ser adotada no Brasil, para a produção do $\mathrm{FeCrAC}$.

\section{METODOLOGIA}

A definição da rota tecnologia mais adequada para a produção do $\mathrm{FeCrAC}$ será baseada nos critérios preconizados pelo BAT - Best Available Technologies (Figura I). Em relação à indústria de não ferrosos, na qual inclui-se o setor de ferroligas, um abrangente estudo foi preparado na virada do milênio e publicado em 200I [5]. Uma versão revisada foi recentemente preparada e publicada em Outubro de 2014, referenciada à IPPC - Industrial Emissions Directive 2010/75/EU [6].

A etapa I consistirá em efetuar um mapeamento das rotas tecnológicas industrialmente existentes para a produção de FeCrAC, segundo o modelo de Beukes et al. [7]. $\mathrm{Na}$ sequência, a etapa 2 tratará de definir a rota mais adequada para a produção do FeCrAC no Brasil. Haverá dois filtros subsequentes e distintos, sendo:

\section{FILTRO I - Avaliação Técnica das Rotas}

A avaliação técnica das rotas será feita a partir de um modelo multicritério de apoio a decisão conforme a seguir:

Os parâmetros de avaliação receberão uma pontuação de I a 5 , sendo que quanto maior o valor, melhor o parâmetro é avaliado. Cada parâmetro receberá um determinado peso, para refletir a importância de cada um deles na avaliação técnica. O resultado final será a multiplicação da pontuação com o peso adotado. Os maiores valores deste resultado indicarão as rotas tecnicamente mais adequadas para a produção de FeCrAC no Brasil.

A definição dos pesos de cada parâmetro será a partir dos resultados da pesquisa realizada em 2009, por 


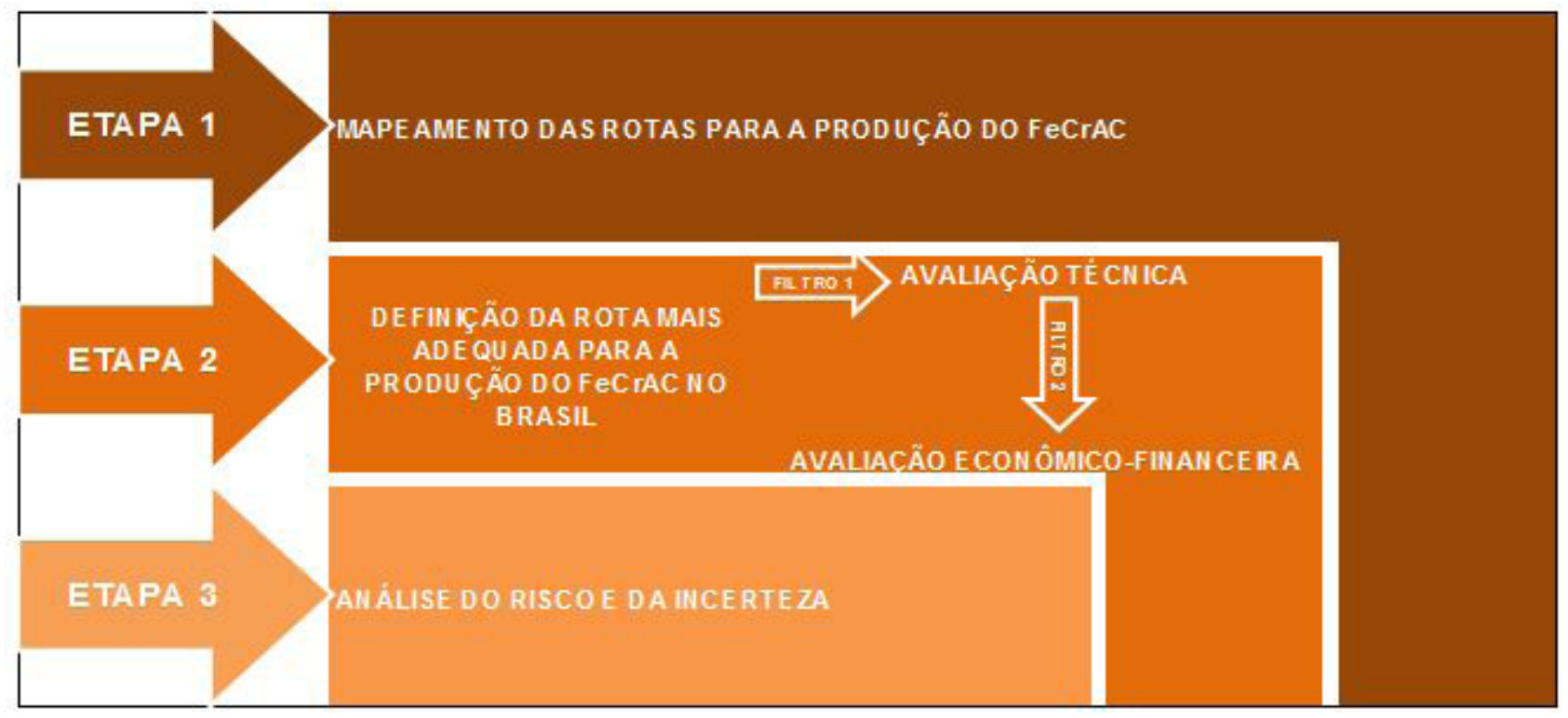

Figura I. Percurso metodológico.

Holappa [8], junto a vários especialistas em ferroligas em vários países. Os parâmetros de avaliação quantitativos e qualitativos que foram utilizados foram propostos por Gasik [3] e JRC [6]. Os critérios e pesos são os seguintes: Eficiência da energia elétrica (peso 5), Eficiência metalúrgica (peso 4), Economia de escala (peso 4), Capital investido (peso 4), Complexidade (peso 4), Meio ambiente e saúde ocupacional (peso 2) e Flexibilidade (peso I).

\section{FILTRO 2 - Avaliação Econômico-financeira}

As rotas selecionadas no primeiro filtro serão submetidas ao segundo e último filtro, que é a avaliação econômico-financeira de projetos mutuamente excludentes.

O fluxo de caixa dos investimentos, capital de giro e critérios para análise do projeto, seguirão a metodologia apresentada por Abreu et al. [9]. O software Microsoft Excel foi usado como ferramenta para os cálculos.

\section{> Fluxo de caixa do projeto:}

O fluxo de caixa será estruturado mediante a estimação dos valores de entradas e saídas de recursos financeiros. No caso base, a taxa de câmbio $(\mathrm{R} \$ / \mathrm{US} \$)$ será a média do período de Janeiro a Setembro de 2015 e será considerado o incentivo fiscal da SUDENE de $75 \%$ redução no imposto de renda para empresas instaladas no nordeste.

Etapas a serem seguidas para estimar o fluxo de caixa:

$\checkmark$ Vida útil do projeto: $O$ horizonte de projeção será de 20 anos.

$\checkmark$ Receita líquida de venda: considera-se que toda a liga produzida será vendida e a receita líquida unitária será o valor praticado pela FERBASA e disponível nas demonstrações financeiras anuais completas de setembro de 2015 .

$\checkmark$ Custos Operacionais Fixos: Serão considerados dados históricos em plantas onde as rotas tecnológicas foram implementadas.

$\checkmark$ Custos Operacionais Variáveis: Os consumos específicos de matérias primas e energia serão calculados a partir de um balanço de massa e térmico. O software Microsoft Excel será usado como ferramenta para os cálculos.

A energia elétrica, no cenário base, será utilizado o preço estimado no mercado livre projetado para novembro de 2015 pela Thymos Energia.

Redutores, quartzo, pasta eletródica e materiais auxiliares serão utilizados os custos incorridos pela FERBASA em setembro de 2015.

Minérios cromo serão também utilizados os custos incorridos pela FERBASA em setembro de 2015.

$\checkmark$ Despesas Operacionais: Adotar-se-á os percentuais em relação à receita líquida, praticados pela FERBASA e disponíveis nas demonstrações financeiras anuais completas de setembro de 2015.

$\checkmark$ Despesa de Depreciação: Será adotado o cálculo do método de depreciação linear, considerando vida útil de 20 anos.

$\checkmark$ Investimento Total: refere-se ao volume comprometido de capital direcionados a geração de resultados futuros. $O$ valor residual do investimento no final do projeto será considerado zero. 
Os investimentos em ativos fixos serão valores a partir do último investimento executado em 2014 para a produção do FeCrAC pela FERBASA e valores estimados pela empresa de engenharia TENOVA.

O investimento em capital de giro será considerado como uma porcentagem do incremento de vendas. A porcentagem atribuída dependerá do giro e do índice de rotação das principais operações do negócio, sendo: clientes igual a 38 dias, estoque igual a 90 dias e fornecedores igual a 25 dias.

A avaliação de projetos de investimentos comumente envolve um conjunto de técnicas que buscam determinar sua viabilidade econômica e financeira, considerando uma determinada Taxa Mínima de Atratividade (TMA), assim definidas por Abreu et al. [9]: Payback simples, Valor presente líquido (VPL), Taxa interna de retorno (TIR), Índice de lucratividade líquida (ILL), Ponto de equilíbrio e Análise de sensibilidade.

Através da análise de sensibilidade, determina-se em que medida um erro ou modificação de uma das variáveis incide nos resultados finais do projeto. A análise de sensibilidade consistirá em calcular os indicadores econômicos em três cenários distintos, conforme a seguir:

$\checkmark$ Cenário I - mais provável: manutenção da quantidade média vendida no mercado interno dos últimos
5 anos (2010 a 20I5), que corresponde a $50 \%$ da capacidade de produção.

$\checkmark$ Cenário 2 - otimista: aumento da demanda no mercado interno em $50 \%$ em relação à média dos últimos 05 anos (2010 a 2015), equivalente, assim, a $75 \%$ da capacidade de produção.

$\checkmark$ Cenário 3 - pessimista: vendas somente para o mercado externo. Este cenário avaliará se o projeto é competitivo em custo para suportar exportar o produto.

\section{RESULTADOS E DISCUSSÃO}

Em geral, quatro bem definidas combinações de processo caracterizam essas rotas tecnológicas [7], conforme mostra a Figura 2.

- ROTA I: Operação em forno elétrico de redução aberto e semifechado, com tratamento dos gases em filtros de manga. Essa é a mais antiga tecnologia, mas ainda é a que predomina no mundo e em vigor no Brasil. Neste tipo de operação minério de cromo lump e finos de minério podem ser fundidos sem prévia aglomeração. Embora tem-se afirmado

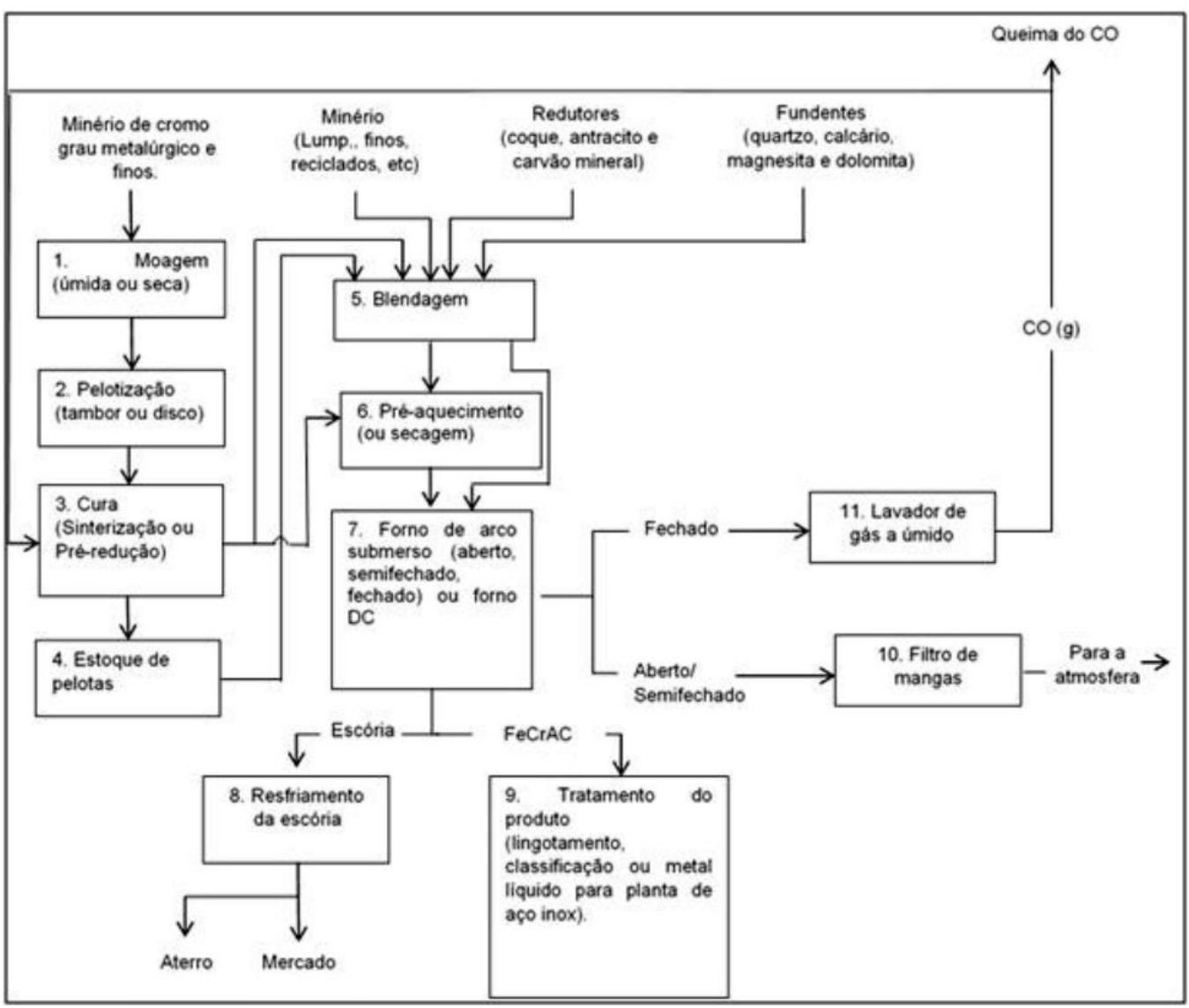

Figura 2. Fluxograma e etapas das rotas tecnológicas para a produção do FeCrAC Fonte: Adaptado de Beukes et al. [7] 
que finos de minérios não podem ser alimentados diretamente no forno a arco submerso sem que isso cause perigosos distúrbios operacionais, uma quantidade substancial de finos de minérios tem sido de fato usados diretamente nos fornos. Tendo como referência o fluxograma da Figura 2, as etapas dos processos são 5, 7, 8, 9 e 10. Alguns fornos abertos e semifechados também podem consumir pelotas, sendo que neste caso devem-se incluir as etapas I, 2, 3 e 4.

- ROTA 2: Operação em forno elétrico fechado, usualmente utilizando minério lump e/ou pelotas sinterizadas, com ou sem pré-aquecimento. Este é o processo mais largamente utilizado na África do Sul e predominante nos projetos de expansão brownfield e greenfield no mundo. Tendo como referência o fluxograma da Figura 2, as etapas do processo normalmente incluem as etapas I, 2, 3, 4, 5, 7, 8, 9 e II, com ou sem a etapa 6. Existe uma forte tendência de todo desenvolvimento de projeto greenfield para $\mathrm{FeCr}$ as etapas 2 (pelotização) e 3 (sinterização), serem integradas com os fornos fechados.

Um exemplo de sucesso de processo integrado com operação de forno elétrico fechado pode ser encontrado no processo de fabricação do $\mathrm{FeCrAC}$ da Outotec, no qual cobre a etapa de aglomeração, utilização do gás do forno e maximização da escala (tamanho) do forno. A planta mais representativa que usa essa tecnologia é a planta da Outokumpu em Tornio, na Finlândia [3].
- ROTA 3: Operação em forno elétrico fechado com alimentação de pelota pré-reduzida. Tendo como referência o fluxograma da Figura 2, as etapas do processo incluem as etapas I , 2, 3, 4, 5, 7, 8, 9, e I I. A pelota alimentada difere substancialmente da pelota sinterizada oxidada, devido ao fato dessas pelotas estar pré-reduzidas e a maioria alimentadas quentes, diretamente no forno fechado, logo após a etapa de pré-redução.

O processo Premus da Xstrata, que opera nas plantas de Lydenburg e Steelpoort na África do Sul, é o único em escala comercial no mundo que utiliza a etapa do processo de pré-redução [3].

> ROTA 4: Operação em forno a arco tipo DC (Direct Current - Corrente Contínua). Para este tipo de operação, a alimentação consiste exclusivamente de materiais finos. Tendo como referência o fluxograma da Figura 2, as etapas do processo incluem as etapas 5, 7 (com forno DC, ao invés do forno a arco submerso), 8, 9 e II. A etapa 6 (secagem) poderia ser incluída.

As rotas mapeadas na primeira etapa foram submetidas a dois filtros subsequentes e distintos, sendo o primeiro filtro a avaliação técnica e o segundo a avalição econômico-financeira. No primeiro filtro foram definidas as rotas mais adequadas tecnicamente para a produção do FeCrAC no Brasil, sem levar em consideração aspectos econômicos. Essa avaliação técnica foi feita pelo próprio

Tabela I. Resultados comparativos para as diferentes rotas de produção do FeCrAC

\begin{tabular}{|c|c|c|c|c|}
\hline & $\begin{array}{c}\text { EFICIÊNCIA } \\
\text { METALÚRGICA }\end{array}$ & $\begin{array}{c}\text { CONSUMO ESPECÍFICO } \\
\text { DE ENERGIA }\end{array}$ & ECONOMIA DE ESCALA & $\begin{array}{l}\text { INDICADOR DE SAÚDE } \\
\text { OCUPACIONAL }\end{array}$ \\
\hline TECNOLÓGICAS & $\begin{array}{c}(\% \mathrm{Cr} \\
\text { recuperado) }\end{array}$ & $(\mathrm{kwh} / \mathrm{t})$ & $\begin{array}{c}\text { (tamanho máximo de um } \\
\text { único forno/ produção de } \\
\text { um único forno) }\end{array}$ & (indexado à geração do $\mathrm{Cr}^{6+}$ ) \\
\hline ROTA I* & $70-75$ & 4.300 & $30 \mathrm{MVA} / 50 \mathrm{kt} / \mathrm{ano}$ & 10 \\
\hline ROTA $2 * *$ & $83-88$ & 3.200 & I 35 MVA / 240 kt/ano & I \\
\hline ROTA 3 & $88-92$ & $2400 * * * *$ & $66 \mathrm{MVA} / 160 \mathrm{kt} / \mathrm{ano}$ & I \\
\hline ROTA 4 & $88-92$ & 4.200 & $60 \mathrm{MW} / \mathrm{I} 10 \mathrm{kt} / \mathrm{ano}$ & I \\
\hline
\end{tabular}

*Sem peneiramento de matéria prima; **Com alimentação de pelotas e com pré-aquecimento da carga; ***Apenas energia elétrica no forno, excluindo-se a energia de combustível associado na etapa de pré redução. Fonte: Adaptado de Gasik [3].

Tabela 2. Pontuação dos parâmetros de avaliação técnica

\begin{tabular}{|c|c|c|c|c|c|}
\hline \multicolumn{6}{|c|}{ AVALIAÇÃO TÉCNICA } \\
\hline CRITÉRIOS & PESO & ROTA I & ROTA 2 & ROTA 3 & ROTA 4 \\
\hline EFICIÊNCIA DA ENERGIA ELÉTRICA & 5 & 2,8 & 3,8 & 5,0 & 2,9 \\
\hline EFICIÊNCIA METALÚRGICA & 4 & 4,0 & 4,8 & 5,0 & 5,0 \\
\hline ECONOMIA DE ESCALA & 4 & $\mathrm{I}, 0$ & 5,0 & 3,3 & 2,3 \\
\hline CAPITAL INVESTIDO & 4 & 5,0 & 3,0 & $\mathrm{I}, 0$ & $\mathrm{I}, 0$ \\
\hline COMPLEXIDADE & 4 & 5,0 & 3,0 & $\mathrm{I}, 0$ & $\mathrm{I}, 0$ \\
\hline MEIO AMBIENTE E SAÚDE OCUPACIONAL & 2 & $\mathrm{I}, 0$ & 5,0 & 5,0 & 5,0 \\
\hline FLEXIBILIDADE & I & 5,0 & 3,0 & $\mathrm{I}, 0$ & 1,0 \\
\hline TOTAL & & 81,2 & 94,8 & 77,3 & 62,5 \\
\hline
\end{tabular}


autor, engenheiro metalurgista, com mais de 25 anos de experiência na indústria de ferroligas no Brasil e no exterior.

A Tabela I mostra os parâmetros quantitativos da avaliação e a Tabela 2 indica a consolidação da pontuação de todos os indicadores.

A partir da avaliação de todos esses parâmetros, as rotas I e 2 é que demonstraram ser tecnicamente mais adequadas para a produção de $\mathrm{FeCrAC}$ no Brasil.

A empresa Ferbasa é detentora de $85 \%$ das reservas brasileiras de minério de cromo. Em março de 20।2, anunciou em fato relevante que 0 montante saltou de 13 milhões para 40 milhões de toneladas do minério Jacurici. Este é o minério destinado para a produção do $\mathrm{FeCrAC}$ no Brasil.

Segundo dados fornecidos pela FERBASA, nos últimos 05 anos a produção do minério Jacurici tem mantido a geração de $80 \%$ de lump e que as pesquisas mais recentes indicam que as reservas existentes deste minério também mantem a geração de lump entre 75 e $80 \%$.

Na rota tecnológica 3, existe a etapa de pré-redução. Para tal, faz-se necessário moer o minério e, a seguir, aglomerá-lo via processo de pelotização. Na rota tecnológica 4 , utiliza-se somente minério fino e apresenta o maior consumo específico de energia elétrica (Tabela I).

O minério Jacurici para a produção do $\mathrm{FeCrAC}$ é predominante lump. Isso implicaria na necessidade de moer este minério, caso fosse utilizar as rotas 3 ou 4.

Ainda não existem estudos aprofundados para validem o desempenho do minério Jacurici nos processos de pelotização e pré-redução, sendo este um dos motivos pelo qual a rota 3 foi descartada.

A análise de viabilidade econômico-financeira (filtro 2) é que definiu, entre as rotas I e 2 , a mais adequada para a produção do FeCrAC no Brasil.
A partir das demonstrações do resultado dos exercícios, foram construídos os fluxos de caixa de cada um dos projetos e, então, foram obtidos os índices econômicos conforme a Tabela 3.

A partir dos resultados apresentados na Tabela 3, foi possível concluir que a rota 2 obteve o melhor resultado em todos os indicadores, sendo assim definida como a rota mais adequada para a produção do $\mathrm{FeCrAC}$ no Brasil.

A partir da Tabela 3, tem-se a seguinte avaliação para a rota 2 :

I. Cria maior valor para o acionista, que é demonstrado pelo maior VPL ( $\mathrm{R} \$ 557.788 .725)$.

2. TIR igual a $24,4 \%$, significando que o fluxo de caixa gerado pelo investimento cobre o custo de capital e "deixa" um excedente de retorno para o investidor.

3. ILL de I,7I, indicando que a cada $R \$ I, 00$ investido, 0 investidor terá $\mathrm{R} \$ 0,7 \mathrm{I}$ de retorno, após ter remunerado o investimento em I4\% a.a. e recuperado o capital investido.

Ao percorrer todas as etapas anteriores, definiu-se a rota 2 como a mais adequada para produção do $\mathrm{FeCrAC}$ no Brasil.

A escolha dos três cenários variando-se a distribuição das quantidades vendidas entre os mercados interno e externo, foi devido ao fato do preço líquido de venda para exportar ser comumente menor que o preço líquido de venda para o mercado interno. Uma breve explicação das características dos cenários está na legenda inferior da Tabela 4.

Conforme é demonstrado na Tabela 4, os indicadores econômicos no cenário 3 , onde toda a produção é exportada,

Tabela 3. Indicadores econômicos das rotas I e 2

\begin{tabular}{lccc}
\hline \multicolumn{1}{c}{ INDICADORES ECONÔMICOS } & UNID. & ROTA I & ROTA 2 \\
\hline TAXA MÍNIMA DE ATRATIVIDADE (TMA) & $\%$ & $14,0 \%$ & $14,0 \%$ \\
VALOR PRESENTE LÍQUIDO (VPL) & R\$ & 173.867 .139 & 557.788 .725 \\
TAXA INTERNA DE RETORNO (TIR) & $\%$ & $17,8 \%$ & $24,4 \%$ \\
ÍNDICE DE LUCRATIVIDADE LíQUIDA (ILL) & - & 1,26 & $1,7 \mid$ \\
PAYBACK SIMPLES (PS) & anos & 5,6 & 4,1 \\
EBITDA (lucro antes de impostos, juros, depreciação e amortização) & $\%$ & $18,8 \%$ & $29,5 \%$ \\
RESULTADO OPERACIONAL LíQUIDO & $\%$ & $10,5 \%$ & $17,1 \%$ \\
\hline
\end{tabular}

Tabela 4. Indicadores econômicos nos cenários I, 2 e 3 para a rota 2

\begin{tabular}{|c|c|c|c|c|}
\hline INDICADORES ECONÔMICOS & UNID. & CENÁRIO I & CENÁRIO 2 & CENÁRIO 3 \\
\hline TAXA MÍNIMA DE ATRATIVIDADE (TMA) & $\%$ & $14 \%$ & $14 \%$ & $14 \%$ \\
\hline PONTO DE EQUILÍBRIO (PE) & $\%$ & $33,1 \%$ & $30,3 \%$ & $41,8 \%$ \\
\hline VALOR PRESENTE LÍQUIDO (VPL) & $\mathrm{R} \$$ & 557.788 .725 & 778.406 .553 & I I6.553.068 \\
\hline TAXA INTERNA DE RETORNO (TIR) & $\%$ & $24,4 \%$ & $28,2 \%$ & $16,3 \%$ \\
\hline ÍNDICE DE LUCRATIVIDADE LÍQUIDA (ILL) & - & I,7I & 1,98 & 1,15 \\
\hline PAYBACK SIMPLES (PS) & anos & 4,1 & 3,6 & 6,0 \\
\hline EBITDA (lucro antes de impostos, juros, depreciação e amortização) & $\%$ & $29,5 \%$ & $32,8 \%$ & $21,7 \%$ \\
\hline RESULTADO OPERACIONAL LÍQUIDO & $\%$ & $17,1 \%$ & $19,4 \%$ & $11,7 \%$ \\
\hline
\end{tabular}


Tabela 5. Indicadores econômicos no 3 para a rota I

\begin{tabular}{lcc}
\hline \multicolumn{1}{c}{ INDICADORES ECONÔMICOS } & UNID. & CENÁRIO 3 \\
\hline TAXA MÍNIMA DE ATRATIVIDADE (TMA) & $\%$ & I $\%$ \\
PONTO DE EQUILÍBRIO (PE) & $\%$ & $69,0 \%$ \\
VALOR PRESENTE LÍQUIDO (VPL) & $\mathrm{R} \$$ & $-267.368 .5 I 7$ \\
TAXA INTERNA DE RETORNO (TIR) & $\%$ & $7,4 \%$ \\
ÍNDICE DE LUCRATIVIDADE LíQUIDA (ILL) & - & 0,59 \\
PAYBACK SIMPLES (PS) & anos & 1,1 \\
EBITDA' & $\%$ & $9,7 \%$ \\
RESULTADO OPERACIONAL LÍQUIDO & $\%$ & $4,3 \%$ \\
\hline
\end{tabular}

' Earnings before interest, tax, depreciation and amortization = lucro antes de impostos, juros, depreciação e amortização.

são positivos. Isso demonstra que a rota 2 é suficientemente competitiva em custo para exportar.

$\mathrm{O}$ preço do $\mathrm{FeCrAC}$ vem apresentando uma tendência de queda nos últimos anos e essa é uma variável que influencia no resultado do projeto. Os cenários I, 2 e 3 admitem, respectivamente, uma redução nesta receita de $14,2 \%$, $18,8 \%$, e $3,4 \%$. Esses são os valores para os quais o VPL é zero em cada cenário. Receitas abaixo desses valores, torna o VPL negativo. Como se esperava, o cenário 3 é o que absorve uma pequena variação no preço do $\mathrm{FeCrAC}$ (vide Tabela 5 -cenário mais desfavorável).

\section{CONCLUSÕES}

Os crescentes preços de energia elétrica, o aumento do rigor das restrições ambientais e as maiores exigências de produtividade vêm reduzindo a competitividade da produção de $\mathrm{FeCrAC}$ baseada na tecnologia atual empregada pela indústria brasileira. Embora alguns avanços já têm sido alcançados na busca de maior competitividade nas ferroligas, este estudo evidenciou que existe uma lacuna para o desenvolvimento de uma metodologia adequada para mapear e definir a rota tecnológica mais adequada para a produção do $\mathrm{FeCrAC}$ no Brasil.

Para definição da rota mais adequada para a produção do $\mathrm{FeCrAC}$, as quatro rotas mapeadas passaram, primeiramente, por um filtro de avaliação técnica. A avaliação econômico-financeira foi o último filtro para definição da rota mais adequada. Neste filtro, a rota 2 apresentou o melhor resultado em todos os indicadores econômicos. Isso foi demonstrado pelo maior VPL, indicando maior criação de valor para o investidor.

Comparando-se com a rota I, a adoção da rota 2 muda drasticamente o patamar de competitividade da indústria de FeCrAC no Brasil:
- A produtividade vai de $942 \mathrm{t} / \mathrm{ano}$.homem para I.864 t/ano.homem;

- Redução em 15\% tanto no custo unitário do produto, como na demanda de energia elétrica;

- Recuperação de energia no processo produtivo;

- Redução no consumo específico de energia elétrica de $4.150 \mathrm{kWh} / \mathrm{t}$ para $3.580 \mathrm{kWh} / \mathrm{t}$. Isso equivale a uma redução anual no custeio com energia elétrica na ordem de $\mathrm{R} \$ 22.944 .395$;

- Redução em mais de $40 \%$ no custo fixo, o que corresponde a uma economia anual na ordem de $\mathrm{R} \$$ 44.438. I08;

- Atendimento as exigências de saúde, segurança e meio ambiente.

Uma das grandes ameaças para a sobrevivência da indústria de $\mathrm{FeCrAC}$ no Brasil é a forte dependência de poucos clientes no mercado interno. A análise do risco e da incerteza mostrou que a rota 2 suporta o cenário 3 pessimista, que é de exportar 100\% da produção. Com isso, mitiga a ameaça de ter poucos clientes no mercado interno e gera a oportunidade de aumento de capacidade, pois o excedente de produção poderia ser exportado.

A indústria tende ser avessa ao risco e raramente opta por tecnologias que ainda não domina totalmente. Este é o caso da indústria de ferroligas brasileira, onde, também, as decisões são extremamente influenciadas pelo custo de investimento e não pelos benefícios dos custos operacionais, ganhos ambientais e na condição de trabalho associados com a tecnologia selecionada. O propósito deste trabalho foi estabelecer uma metodologia que possa aumentar a credibilidade e a sensibilização para a adoção dessas rotas tecnológicas.

\section{REFERÊNCIAS}

I Tolmasquim MT, Szklo ASA. matriz energética brasileira na virada do milênio. Rio de Janeiro: ENERGE-COPPE/ UFRJ; 2000.

2 Leite AAF, Bajay SV, Gorla FD. Oportunidades de eficiência energética para a Indústria: relatório setorial: setor de ferroligas. Brasília: CNI; 2010. 
3 Gasik M. Handbook of ferroalloys: theory and technology. Oxford: Elsevier; 2013.

4 Martins GJ. 2004. A metodology to identify, quantify and verifiy cost benefits of energy and process improvement opportunities in a ferro-metal production plant [dissertação de mestrad]. Potchefstroom: School of Mechanical and Materials Engineering at the North-West University; 2004.

5 Joint Research Centre - JRC. Best available techniques (BAT) reference document for the non-ferrous metals industries. Seville; 200 I [acesso em I0 ago. 20I5]. Disponível em: http://eippcb.jrc.ec.europa.eu

6 Joint Research Centre - JRC. Best available techniques (BAT) reference document for the non-ferrous metals industries. Seville; 2014 [acesso em 10 ago. 20I5]. Disponível em: http://eippcb.jrc.ec.europa.eu

7 Beukes, J.P., Dawson, N.F; Zyl, P.G. Theoritical and practical aspects of $\mathrm{Cr}(\mathrm{VI})$ in the South African ferrochrome industry. In: INFACON XII; 2010; Helsink. Helsink; 20I0. p. 53-62.

8 Holappa, L. Towards sustainability in ferroalloys production. In: INFACON XII; 20I0; Helsink. Helsink; 20I0. p. I-I0.

9 Abreu JCF Fo, Souza CP, Gonçalves DA, Cury MVQI. Finanças corporativas. Rio de Janeiro: FGV, 2003.

Recebido em: 20 Jun. 2016

Aceito em: 14 Set. 2016 